\title{
$\ell$-space spectroscopy of the Cosmic Microwave Background with the BOOMERanG experiment
}

P. de Bernardis ${ }^{1}$, P.A.R. Ade ${ }^{2}$, J.J. Bock ${ }^{3}$, J.R. Bond ${ }^{4}$, J. Borrill ${ }^{5}$, A. Boscaleri ${ }^{6}$, K. Coble ${ }^{7}$, C.R. Contaldi ${ }^{4}$, B.P. Crill ${ }^{8}$, G. De Gasperis ${ }^{9}$, G. De Troia ${ }^{1}$, P. Farese ${ }^{7}$, K. Ganga ${ }^{10}$, M. Giacometti ${ }^{1}$, E. Hivon ${ }^{10}$, V.V. Hristov ${ }^{8}$, A. Iacoangeli ${ }^{1}$, A.H. Jaffe ${ }^{11}$, W.C. Jones ${ }^{8}$, A.E. Lange ${ }^{8}$, L. Martinis ${ }^{12}$, S. Masi ${ }^{1}$, P. Mason ${ }^{8}$, P.D. Mauskopf ${ }^{13}$, A. Melchiorri ${ }^{14}$, P. Natoli ${ }^{9}$, T. Montroy $^{7}$, C.B. Netterfield ${ }^{15}$, E. Pascale ${ }^{6}$, F. Piacentini ${ }^{1}$, D. Pogosyan ${ }^{4}$, G. Polenta ${ }^{1}$, F. Pongetti ${ }^{16}$, S. Prunet $^{4}$, G. Romeo ${ }^{16}$, J.E. Ruhl ${ }^{7}$, F. Scaramuzzi ${ }^{12}$, N. Vittorio ${ }^{14}$

${ }^{1}$ Dipartimento di Fisica, Universitá La Sapienza, Roma, P.le A. Moro, 2, 00185, Italy. ${ }^{2}$ Queen Mary and Westfield College, London, UK. ${ }^{3}$ Jet Propulsion Laboratory, Pasadena, CA, USA. ${ }^{4}$ C.I.T.A., University of Toronto, Canada. ${ }^{5}$ N.E.R.S.C., LBNL, Berkeley, CA, USA. ${ }^{6}$ IROE-CNR, Firenze, Italy. ${ }^{7}$ Dept. of Physics, Univ. of California, Santa Barbara, CA, USA. ${ }^{8}$ California Institute of Technology, Pasadena, CA, USA. ${ }^{9}$ Department of Physics, Second University of Rome, Italy. ${ }^{10}$ IPAC, Caltech, Pasadena, CA, USA. ${ }^{11}$ Department of

Astronomy, Space Sciences Lab and Center for Particle Astrophysics, University of CA, Berkeley, CA 94720 USA. ${ }^{12}$ ENEA, Frascati, Italy. ${ }^{13}$ Dept. of Physics and Astronomy, Cardiff University, Cardiff CF24 3YB, Wales, UK. ${ }^{14}$ Nuclear and Astrophysics Laboratory, University of Oxford, Keble Road, Oxford, OX $3 R H$, UK. ${ }^{15}$ Depts. of Physics and Astronomy, University of Toronto, Canada. ${ }^{16}$ Istituto Nazionale di Geofisica, Roma, Italy.

Abstract. The BOOMERanG experiment has recently produced detailed maps of the Cosmic Microwave Background, where sub-horizon structures are resolved with good signal to noise ratio. A power spectrum (spherical harmonics) analysis of the maps detects three peaks, at multipoles $\ell=\left(213_{-13}^{+10}\right),\left(541_{-32}^{+20}\right),\left(845_{-25}^{+12}\right)$. In this paper we discuss the data analysis and the implications of these results for cosmology.

\section{INTRODUCTION}

The Cosmic Microwave Background (CMB) is a window on the Early Universe. It comes from an epoch when the age of the Universe was a few hundred thousand years (50000 times younger than today), the temperature was about 3000 $\mathrm{K}$ (1000 times more than today) and the density was one billion times larger than today [1],[2]. The very presence of the CMB is a compelling proof of the existence of a hot and dense initial phase in the Universe [3], [4]. The physics of the photons-baryons plasma present at that epoch, its interaction with the underlying dark matter distribution, and the resulting observable effects on the CMB have been studied in great detail [5].

If the inflationary scenario is true, photons coming from that epoch carry information which has been encoded at much earlier epochs, thus enabling us to investigate the history of the Universe as early as $10^{-36} \mathrm{~s}$ after the Big-Bang. An image of the CMB is a (processed) image of quantum fluctuations present in the Universe before the inflation phase [6], at energies of the order of the GUT energies.

In this framework, the statistical properties of the image of the CMB $\Delta T(\alpha, \delta)$ can be derived from first principles, given a small set of cosmological parameters. $\Delta T(\alpha, \delta)$, is expected to be a 2-D random gaussian field, with statistical properties fully described by its power spectrum $c_{\ell}$. Here $c_{\ell}=\left\langle\left|a_{\ell, m}\right|^{2}\right\rangle$, where $\Delta T(\alpha, \delta)=\sum_{\ell, m} a_{\ell, m} Y_{m}^{\ell}(\alpha, \delta)$. Analyzing the image and its power spectrum, it is then possible to estimate the cosmological parameters [7].

Many experimental teams have actively worked on the measurement of the spectrum, of the anisotropy power spectrum and of the polarization of the CMB. The purely Planckian nature of the spectrum has been established by 
the FIRAS spectrometer on board of the COBE satellite [8]. It is the proof of the cosmological nature of the CMB and of the Hot Big Bang theory proposed by Gamow in the 50s. The intrinsic, faint large scale anisotropy has been first detected by the DMR instrument on board of the COBE satellite [9]. Its low level and its power spectrum supported the inflationary hypothesis [6]. The degree and sub-degree-scale anisotropy has been detected by several ground based and balloon-borne experiments. Only recently, however, it has been possible to first detect the presence of peaks in the power spectrum [10], [11], [12], and to produce images where the sub-degree anisotropy is clearly visible [13], [14], [15], [16]. The presence of multiple peaks is the confirmation of the presence of acoustic oscillations in the plasma before recombination [5] and allows the detection of several important cosmological parameters [17], [18], [33].

In this paper we report results from the BOOMERanG experiment, a balloon-borne microwave telescope with cryogenic bolometric detectors. Several aspects of the instrument and other results are described in companion papers in this same conference proceedings [19], [20], [21], [22], [23], [24]: here we focus on the analysis techniques and on the cosmological significance of the results.

\section{$\ell$-SPACE SPECTROSCOPY}

The detection of structures in the CMB is a difficult experimental problem. The size of the observable temperature fluctuations is of the order of a few tens $\mu K$, while instrumental, local and astrophysical backgrounds can be as large as few $K$. The differences in spectral and angular distributions allow the experimentalists to separate the cosmological component from the contaminations, but elaborate modulation techniques are needed [25], [19],[26]. Interferometers directly sample the correlation function of the temperature fluctuations [27], while total power receivers sample the temperature map: the power spectrum is derived by first organizing the time-ordered observations in a map and then performing the harmonic analysis.

In the case of BOOMERanG modulation is achieved by scanning the sky at constant elevation and constant azimuth speed. The signal from the detector is AC coupled and high pass filtered. Since most of the contaminating signals are either constant or smooth in the sky, they are efficiently rejected by this modulation. The disadvantage of this technique is that it results in an anisotropic filtering of the sky maps, which has to be taken properly in account in the data analysis (see below).

A further level of modulation comes from sky rotation. In fact, the central azimuth of the azimuth scans tracks the azimuth of the best sky region, while elevation is not changed during the scan, and is only changed in steps every several hours. The result of this strategy is that, due to sky rotation, the scans are gradually tilted in the sky, and the same pixel will be re-observed during the same day in differently tilted scans. This produces significant crosslinking in the sky coverage, which is important for the map-making algorithm used to create the image of the sky. The same process is repeated for several days. The comparison of maps obtained in different days, when the payload has drifted by thousands of $\mathrm{km}$ and the ground configuration is completely different, is a very effective tool to exclude contamination of the sky maps coming from the telescope sidelobes.

The peak to peak length of our azimuth scans is $\Delta A \sim 60^{\circ}$, so that the scans in the sky have a length $\Delta \theta \sim \Delta A \cos e \sim$ $42^{\circ}$. This length has been selected as the best compromise between several factors: sky coverage, avoidance of the sun, repetition frequency of scans, detector's speed, $1 / \mathrm{f}$ knee in detectors noise, etc. As a result, our $\ell$-space resolution is limited to $\Delta \ell \sim \pi / \Delta \theta \sim 4$. This is more than enough to resolve the acoustic peaks present in the power spectrum of the CMB anisotropy, which have a width $\Delta \ell_{p} \sim 100$ [5]. In practice, we degrade the instrumental resolution to $\sim \Delta \ell_{p} / 2$ by binning in $\ell$, in order to improve the signal to noise ratio in each $\ell$ bin. The estimates of the power spectrum averaged over wide $\ell$ bins are called bandpowers. The finite length of the scans also limits the lowest multipole detectable $\ell_{\min } \sim \Delta \ell$, but in practice a much higher $\ell_{\min } \sim 25$ is set by the presence of drifts and $1 / f$ noise. The maximum multipole observable in constant speed scans depends on the angular resolution of the telescope, on the time response of the detector [28] and on its noise; in general the sensitivity of the instrument to different multipoles is described by a suitable window function taking into account these effects (see below). In the case of BOOMERanG $\ell_{\max } \lesssim 1200$.

\section{THE 1998/99 LDB DATA}

The BOOMERanG payload was flown by NASA-NSBF on Dec.29, 1998, from McMurdo (Antarctica). It remained at float for 10.6 days, circumnavigating Antarctica at an average altitude of $37 \mathrm{~km}$. About 57 million 16-bit samples of the signal were collected for each of the 16 detectors. The data were edited for known instrument glitches, temperature 
fluctuations, and cosmic rays events. Less than $5 \%$ of the bolometer data has been found to be contaminated. Constrained realizations of noise were substituted to the contaminated signals.

The pointing has been reconstructed from the signals of the laser gyroscopes, of the differential GPS, and of the sun sensors. In the most recent pointing solution, repeated observations of compact sources show that the accuracy of the reconstruction is $\lesssim 2.5$ arcmin $r m s$. Random errors in the pointing have the effect to smear-out the signals from small sources. This adds in quadrature to the intrinsic angular resolution of the telescope (9.5' FWHM at $150 \mathrm{GHz}$ ). The finite size of the pixelization has a similar effect. In $\ell$ space these three effects are modelled by the low pass filter $W(\ell)$ shown in fig.1 (called window function), which has to be taken into account in the reconstruction of the angular power spectrum of the sky. It is evident how these effects limit the sensitivity of our observations at high multipoles.

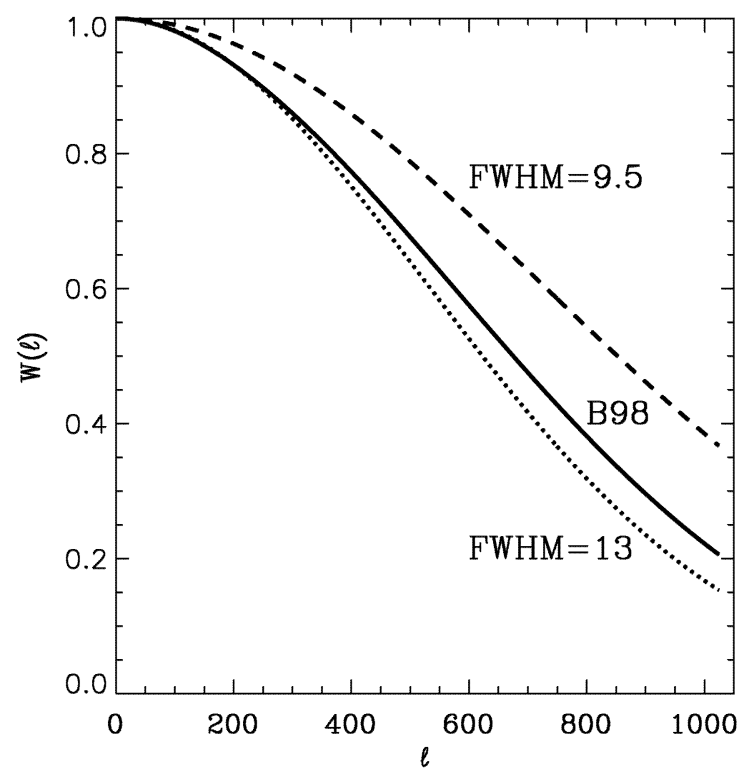

FIGURE 1. Window function $W(\ell)$ for the BOOMERanG instrument (B98) and for gaussian beams with FWHM=9.5' and 13'.

The time-domain high-pass filter is not included in the window function of fig.1 since its effect is, in general, anisotropic. It can be shown, however, that for our particular scan strategy the time-domain high pass filter acts as a high pass filter in the multipoles domain [29].

Sky maps have been constructed from the time ordered data and pointing using four independent methods: naive maps (just coadding data on the same pixel); maximum likelihood maps obtained using the MADCAP package ([30]); maximum likelihood maps obtained using the iterative method of [31]; suboptimal maps obtained using the fast map making method of [29].

All methods produce very similar maps. In fig. 2 we show the central region of the $150 \mathrm{GHz}$ map from the B150A channel obtained with the iterative method [31]. The color code used in the BOOMERanG maps correspond to temperature fluctuations of a $2.73 \mathrm{~K}$ blackbody.

Degree-scale structures with amplitude of the order of $100 \mu K$ are evident in the map at $150 \mathrm{GHz}$. Consistent structure is also evident in the maps at 90 and $240 \mathrm{GHz}$. The similarity of the temperature maps obtained at different frequencies [13] is the best evidence for the CMB origin of the detected fluctuations. Foregrounds contamination can be constrained significantly in the center of the observed sky region [13], [20].

\section{THE POWER SPECTRUM}

Netterfield et al. [15] have computed the power spectrum of the central region of the BOOMERanG 150GHz maps ( $1.8 \%$ of the sky). The result has been obtained with a monte-carlo technique, which allows to estimate effectively the effects of sky coverage, anisotropic filtering, system noise and beam on the measured power spectrum [29]. Using about 10 times more data and about $80 \%$ more sky than the original data release, with an improved pointing solution 


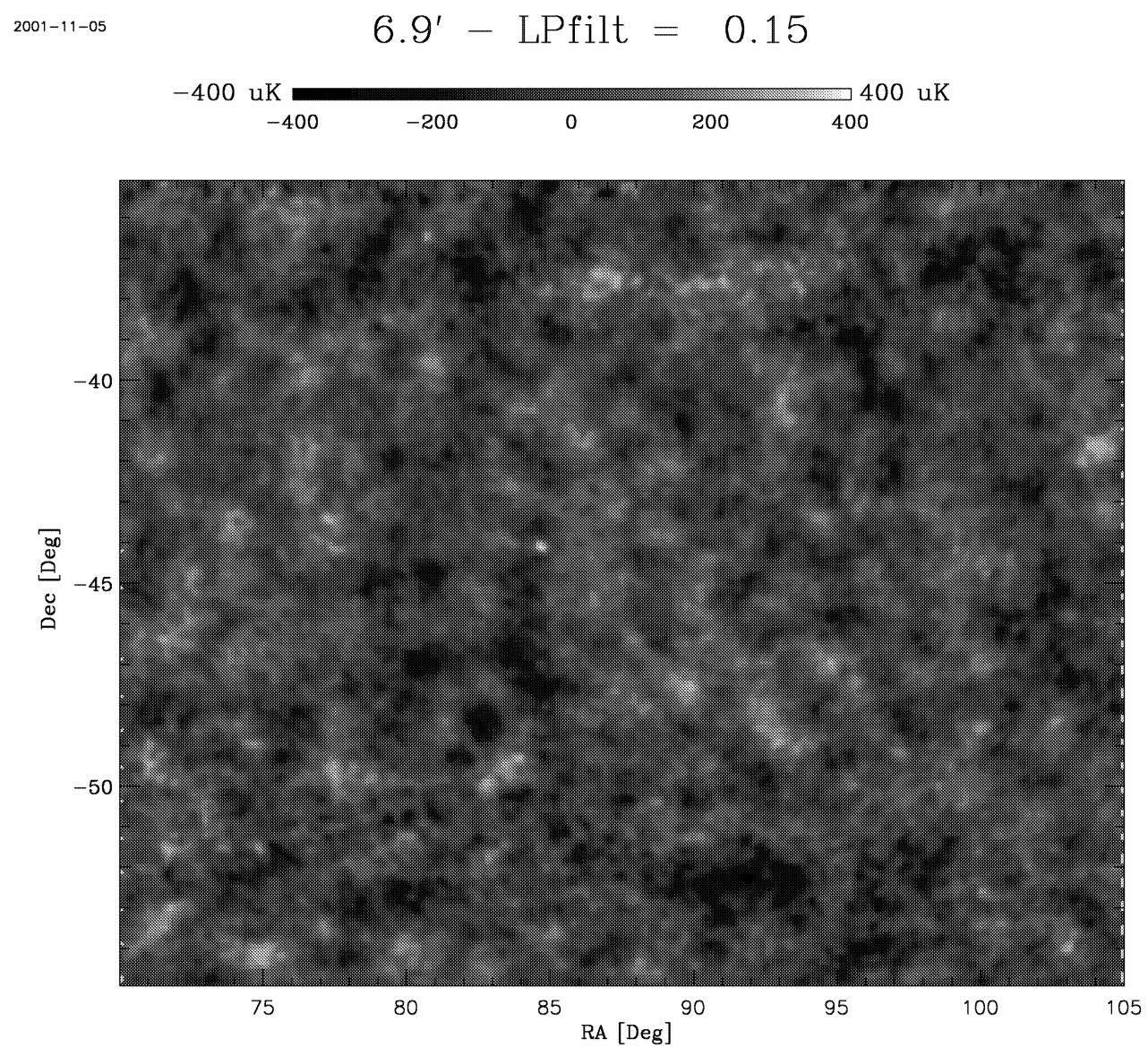

FIGURE 2. Map of the microwave sky measured by BOOMERanG at $150 \mathrm{GHz}$. The resolution of the observations is 12 arcmin. Healpix pixelization with $3.5^{\prime}$ pixel side has been used. The units are for thermodynamic temperature fluctuations of a $2.73 \mathrm{~K}$ blackbody. The same structures are visible in the $90 \mathrm{GHz}$ and in the $240 \mathrm{GHz}$ maps of BOOMERanG.

and a better measurement of the effective beam, it was possible to detect three peaks in the power spectrum of the CMB (see fig.3). Bandpowers have been computed following the recipe of [50]. These data are compared to the simultaneous data releases of the DASI [32] and MAXIMA [33] teams in fig.4.

Given the orthogonality of the experimental and analysis methods, the agreement of the three results is very good, at least visually. The existing anti-correlations in the bandpowers, and the presence of some overlap in the sky coverage of the BOOMERanG and DASI data should be taken into account for a more quantitative comparison. Such a comparison will be the best argument to exclude significant systematic effects in the three spectra.

\section{COSMOLOGY-INDEPENDENT DATA ANALYSIS}

Let's consider first the problem of assessing the statistical properties of the image of the sky we have obtained at $150 \mathrm{GHz}$. We are interested to see if the temperature of the CMB in the sky is distributed as a Gaussian. If this is true, then the power spectrum measures all the information encoded in the image. The gaussianity of the image is not a trivial result of the central limit theorem. As a matter of facts, images of the sky with the same resolution, but at higher frequencies, are highly non-gaussian. A visual exam of the IRAS maps of interstellar dust emission at 100 $\mu m$ is very convincing in this regard [34]. In fig.5 we compare the 1-P distribution of the 150GHz map (dominated 


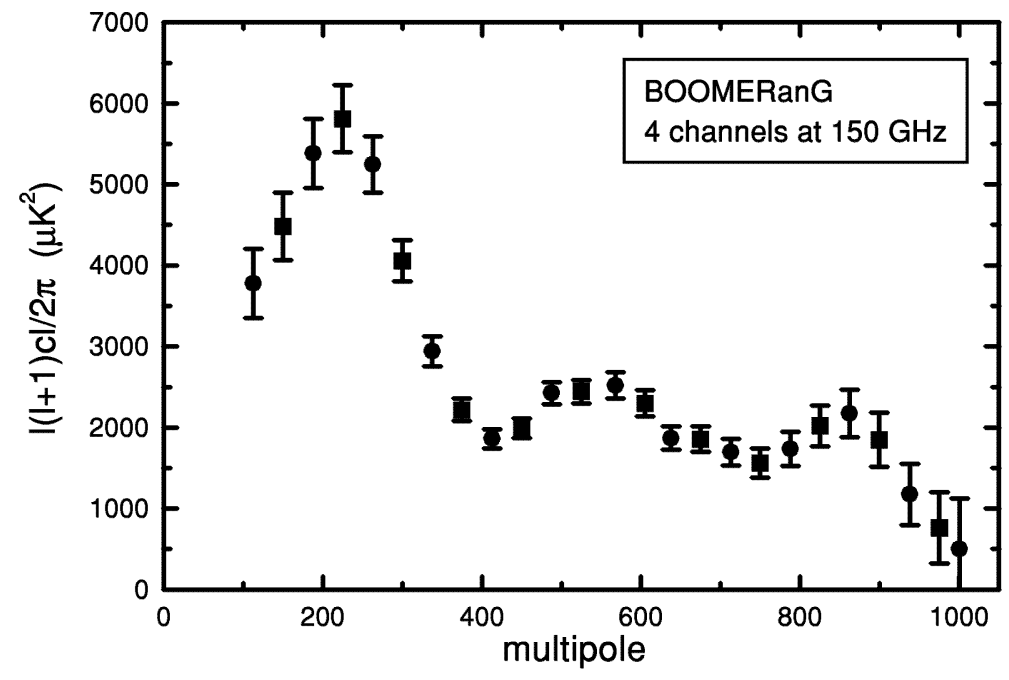

FIGURE 3. CMB anisotropy power spectrum detected by BOOMERanG. The dataset plotted as circles and the dataset plotted as squares are significantly correlated, and are both plotted to show the effect of different binning in $\ell$-space. The different bandpowers of the same dataset are instead effectively uncorrelated. The gain $(10 \%$ at $1 \sigma)$ and beam $\left(1.4^{\prime}\right.$ at $\left.1 \sigma\right)$ calibration errors are not plotted, since they are totally correlated for all the bandpowers.

by CMB anisotropy) to the 1-point distribution of the same patch of the sky measured by BOOMERanG at $410 \mathrm{GHz}$ (dominated by thermal emission of interstellar dust). Even this naive test shows that the CMB is something special, being very accurately gaussian distributed. Of course, for a more quantitative test of the 1-P distribution it is necessary to take into account the correlation properties of the signal and of the noise in the data.

The simplest non-gaussianity estimators in the pixel space are Skewness and Kurtosis of the 1-point distribution, and the Minkowski functionals. [35] have analyzed the $150 \mathrm{GHz}$ maps of BOOMERanG using these five estimators, and a Monte-Carlo approach to account for the correlations in the data, to compute the statistical significance of the results and assess the effect of systematics. All the tests are consistent with the gaussian hypothesis, as reported in table 1. Small non gaussian signals added to the gaussian CMB fluctuations can be excluded with different levels of significance, depending on the nature of the contaminants. For example the rms of fluctuations distributed as a $1 \mathrm{DOF}$ $\chi^{2}$ must be less than $3 \%$ of the rms of the CMB. Fluctuations due to instrumental effects, as well as fluctuations due to the dust foreground and to extragalactic point sources are found to be irrelevant. Spectral methods to study gaussianity are also being exploited. There are many kinds of non-gaussianity [36], and a through analysis will require the use of many different methods.

Given the results above, however, it is reasonable to assume that the power spectrum is the only tool we need to study the statistical properties of the image. [15] have discussed how the measured power spectrum of the sky is robust against variations of the $\ell$-binning, channel selection, data subset selection, effects of uncertainties in the beam and effects of the noise. [17] have shown that the three peaks and two dips present in the power spectrum are statistically significant. The first peak is at $\ell_{1}=\left(213_{-13}^{+10}\right)$ (the errors correspond to a $1 \sigma$ confidence interval in the location if the peak). Its amplitude is detected at $\gtrsim 5 \sigma$, while for the second peak $\left(\right.$ at $\left.\ell_{2}=\left(541_{-32}^{+20}\right)\right)$ and third peak $\left(\right.$ at $\ell_{3}=\left(845_{-25}^{+12}\right)$ ) the amplitudes are detected at basically $2 \sigma$. Several methods to measure the location and amplitude of the peaks have been compared, all producing very consistent results. In particular, the results of fits using empirical functions are consistent with the results of fits using a database of adiabatic inflationary spectra of the CMB [17]. 


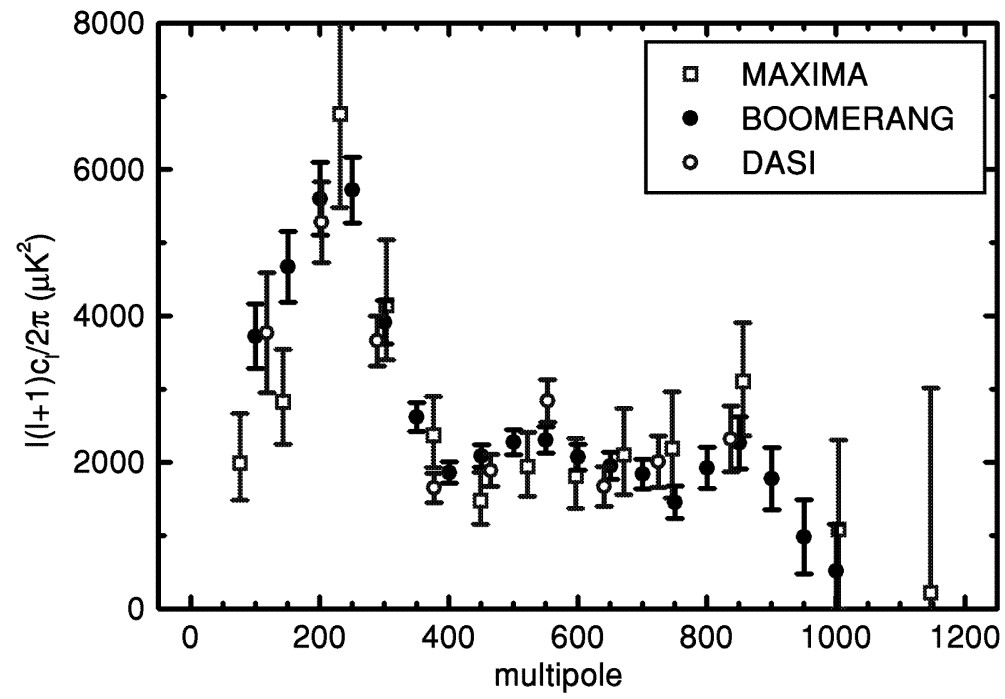

FIGURE 4. CMB anisotropy power spectrum detected by BOOMERanG, MAXIMA and DASI. Approximately uncorrelated bandpowers are plotted for each of the experiments. The error bars represent statistical errors only.

\section{COSMOLOGICAL SIGNIFICANCE AND PARAMETERS ESTIMATION}

The presence of "acoustic" features in the power spectrum of the CMB has been forecast long time ago [37],[38]. In the hot big bang model, it is the result of acoustic waves present in the pre-recombination Universe, and of their behaviour inside the acoustic horizon, in the presence of fluctuations in the density of the dominating form of matter. The same density fluctuations which are sources of the acoustic oscillations in the pre-recombination plasma are also responsible for the gravitational collapse starting after recombination, and leading to the formation of the large scale structures we see in the nearby Universe. Wiggles seem to be present in the spectrum of the large scale distribution of galaxies detected by the recent $2 \mathrm{dF}$ survey [39]; if this will be confirmed and if the wiggles will be found to be consistent with the modulation of the transfer function produced by "acoustic" features, it will be a wonderful success of this theory.

The alternative model of formation of galaxies from topological defects should lead to non gaussian CMB anisotropies [40], and fails to reproduce the observed power spectrum [41]: it can only be subdominant [42].

A large amount of work has been spent to accurately predict the power spectrum of the CMB in the adiabatic inflationary scenario. As of today, detailed fast codes are available [43], [44], and can be used to setup large databases of power spectra, to which the measured spectrum can be compared. More work is still to be done to properly include in the analysis the possible existence of isocurvature modes [45].

In this framework, the location of the first peak in the power spectrum mainly depends on the curvature of the Universe. The first peak is due to those fluctuations that enter the horizon shortly before recombination, and have just enough time to fully compress before recombination happens. The size of these perturbations is thus very similar to the size of the acoustic horizon at recombination. We thus have a "standard ruler", a few hundred thousand light years long, placed at a distance of about 14 billion light years. If we take into account the fact that the Universe has expanded by a factor $\sim 1000$ since recombination, we conclude that these fluctuations should appear as $\sim 1^{o}$ spots in the CMB. This is correct only if the geometry of the Universe is Euclidean, not curved, i.e. if the average mass-energy density of the Universe is the critical one $(\Omega=1)$. If, instead, the mass-energy density is higher than critical $(\Omega>1)$, the geometry of space will have a positive curvature and the photons will travel along curved geodesics. The excess density will act as a magnifying glass, and the same fluctuations in the CMB will appear as spots larger than $1^{o}$. The opposite will happen if the density is lower than critical, acting as a de-magnifying glass and producing a typical angular size of the 

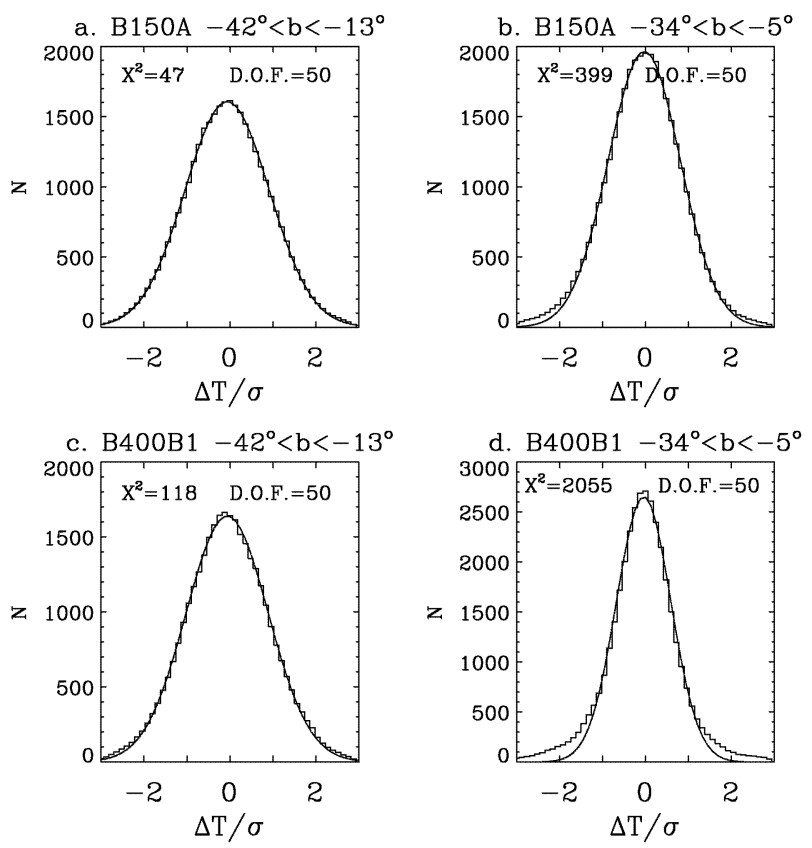

FIGURE 5. In the top row, we plot the 1-P distribution of the ratio $\Delta T_{i} / \sqrt{\sigma_{i}^{2}+\sigma_{s k y}^{2}}$ for the $150 \mathrm{GHz}$ map (dominated by CMB fluctuations) in a box at high galactic latitude (a) and in a box at intermediate galactic latitudes (b). In the lower row the same distributions are plotted for the map at $410 \mathrm{GHz}$, which is dominated by interstellar dust

fluctuations smaller than $1^{\circ}$. In any case the presence of a typical size of the fluctuations $\theta$ will produce a peak in the power spectrum of the $\mathrm{CMB}$ at $\ell_{1}=\pi / \theta$. By measuring the location of the peak it will thus be possible to measure $\Omega$. The quantitative treatment of this angular-size vs distance test can be found in [46], [47]. In general, $\ell_{1}$ decreases when $\Omega$ increases, but the location of the first peak is also controlled by $\Omega_{\Lambda}$. Only if $\Omega_{\Lambda}=0$ the simple relationship $\ell_{p} \sim \Omega^{-\frac{1}{2}}$ holds. The measurement of $\ell_{1}$ from the BOOMERanG power spectrum is $\ell_{1}=\left(213_{-13}^{+10}\right)$, which is consistent with a flat geometry of the Universe. Rigorous confidence intervals for the parameter $\Omega$ can be found with the Bayesian analysis of the full power spectrum as described below; frequentist methods are also being developed [48].

The ratio between the amplitude of the second peak and the amplitude of the first one depends mainly on the physical density of baryons $\Omega_{b} h^{2}$ and on the tilt of the density fluctuations spectrum. A high density of baryons favors compressions against rarefactions: the odd-order peaks (compression) are enhanced while the even-order peaks (rarefaction) are depleted. From the BOOMERanG power spectrum the ratio is $(5450 \pm 350) /(2220 \pm 330)=$ $(2.45 \pm 0.52)$. Assuming a scale invariant power spectrum of the density fluctuations $(n=1)$, this corresponds to a physical density $\Omega_{b} h^{2} \sim 0.02$. Again, better constraints are found by means of the Bayesian analysis of the full power spectrum as described below. In fact, a tilt of the density fluctuations spectrum $(n<1)$ has the same effect of a high baryons density in depleting the second peak with respect to the first one, so there is a degeneracy between the two parameters. But the effects of the two quantities on the amplitude of the third peak are different. The amplitude of the third peak is increased by a high baryon density, while it is decreased by a red $(n<1)$ primordial density fluctuation spectrum. The result is that extending the observations to $\ell \sim 1000$ breaks the degeneracy between $n$ and $\Omega_{b} h^{2}$, thus allowing a determination of both the parameters.

It is important to stress the fact that our result for $\Omega_{b} h^{2}$ agrees with the constraint on $\Omega_{b} h^{2}$ from the Big Bang Nucleosynthesis. In fact, the physical density of baryons affects the yield of the nuclear reactions happening in the first few minutes after the big bang. The resulting primordial abundances of light elements are measured by the optical absorption spectra of primordial clouds of matter [49]. It is evident that both the physics and the experimental methods involved in these two measurements of $\Omega_{b} h^{2}$ are completely orthogonal to the CMB ones. The fact that the two estimates of $\Omega_{b} h^{2}$ agree so well should be considered a great success of the Hot Big Bang model.

The multiple peaks and dips are a strong prediction of the simplest adiabatic inflationary models, and more generally of models with passive, coherent perturbations. Although the main effect giving rise to them is regular sound compression and rarefaction of the photon-baryon plasma at photon decoupling, there are a number of influences that 
make the regularity only roughly true. The best way to extract all the information encoded in the data is by comparison to a large database of $C_{\ell}$ spectra. In order to limit the size of the database, we considered for the first approach the class of adiabatic inflationary models. We have explored a parameter space with 6 discrete parameters and a continuous one. The parameters ranged as follows: $\Omega_{m}=0.11, \ldots, 1.085$, in steps of $0.025 ; \Omega_{b}=0.015, \ldots, 0.20$, in steps of $0.015 ; \Omega_{\Lambda}=0.0, \ldots, 0.975$, in steps of $0.025 ; h=0.25, \ldots, 0.95$, in steps of 0.05 ; spectral index of the primordial density perturbations $n_{s}=0.50, \ldots, 1.50$, in steps of $0.02, \tau_{C}=0 ., . ., 0.5$, in steps of 0.1 . The overall amplitude $C_{10}$, expressed in units of $C_{10}^{C O B E}$, is allowed to vary continuously. We used the BOOMERANG power spectrum expressed as 18 bandpowers $C_{b}$ [15] and we computed the likelihood for the cosmological model $C_{b}^{T}$ as $\exp \left(-\chi^{2} / 2\right)$, where $\chi^{2}=\left(C_{b}-C_{b}^{T}\right) M_{b b^{\prime}}^{-1}\left(C_{b^{\prime}}-C_{b^{\prime}}^{T}\right)$. Here $M_{b b^{\prime}}$ is the covariance matrix of the measured bandpowers; $C_{b}^{T}$ is an appropriate band average of $C_{\ell}$. A $10 \%$ Gaussian-distributed calibration error in the gain and a 1.4' (13\%) beam uncertainty were included in the analysis as additional parameters with gaussian priors. The COBE-DMR bandpowers used were those of [50], obtained from the RADPACK distribution [51]. The 95\% confidence intervals for the parameters we find in this way depend to some extent on the priors assumed. Using COBE and BOOMERanG data only, with a weak prior $0.45<h<0.90$, significantly constrains three parameters: $0.9<\Omega<1.15,0.8<n<1.1$ and $0.015<\Omega_{b} h^{2}<0.029$. Using more restrictive priors, deriving from the properties of the large scale distribution of Galaxies $\left(\sigma_{8}\right.$ and $\left.\Gamma\right)$, or the data of high-redshift supernovae, or the measurement of $h$ by the HST, produces narrower, consistent intervals for these parameters [15], [17]. This fact suggests a good overall consistency of the present cosmological paradigm. Including these priors, it is also possible to constrain the two additional forms of mass-energy contributing to the total mass-energy density in the Universe, i.e. dark matter and dark energy. We find that the $95 \%$ confidence intervals for $\Omega_{b} h^{2}$ and $\Omega_{\Lambda}$ are $0.36<\Omega_{\Lambda}<0.72$ and $0.09<\Omega_{b} h^{2}<0.18$ ( LSS prior); $0.52<\Omega_{\Lambda}<0.88$ and $0.01<\Omega_{b} h^{2}<0.17$ (SN1a prior); $0.40<\Omega_{\Lambda}<0.84$ and $0.06<\Omega_{b} h^{2}<0.26$ (HST $h$ prior). The detection of a non-zero $\Omega_{\Lambda}$ comes thus from independent paths and sets a formidable challenge to our understanding of fundamental physics [52].

\section{CONCLUSIONS}

The BOOMERanG experiment has produced multi-frequency maps of the microwave sky, where the structure of the CMB has been resolved with high signal to noise ratio. The structures in the CMB are gaussian, and their power spectrum features three peaks. This is consistent with the presence of acoustic oscillations in the primeval plasma. It also fits the predictions of the adiabatic inflationary scenario. The values of the cosmological parameters inferred in this scenario point to a flat universe with nearly scale-invariant initial adiabatic perturbations and a significant contribution of dark energy to the total density of the Universe.

Significant work remains to be done with the BOOMERanG data. We are working to analyze all the remaining channels sensitive to the CMB for a more accurate determination of the power spectrum. In parallel, we are currently assessing the gaussianity of the maps with different methods. Also, we are using the observations of Galactic sources in order to improve the gain and beam calibration accuracy. A search for Sunyaev-Zeldovich effect in the direction of the rich clusters of Galaxies present in the observed region is also being carried out. Methods for components separation and the data of the higher frequency channel are being used to investigate the properties of interstellar cirrus clouds.

\section{ACKNOWLEDGMENTS}

This activity has been supported by the University of Rome La Sapienza, Programma Nazionale di Ricerche in Antartide and Agenzia Spaziale Italiana in Italy, by NASA and NSF in USA, by PPARC in UK and by Univ. of Toronto in Canada.

\section{REFERENCES}

1. Weinberg S., 1977, The first three minutes, Basic Books, ISBN-0465024378

2. Peebles P.J.E, 1994, Principles of Physical Cosmology, Princeton Series in Physics

3. Gamow G., 1946, Phys.Rev., 70, 572

4. Dicke R.H., et al., 1965, Ap.J., 142, 414

5. Hu W., Sugiyama N. \& Silk J., 1997, Nature, 386, 37

6. Kolb E.W. and Turner M.S, 1990, The Early Universe, Addison-Wesley 
7. see e.g. Efstathiou G., and Bond, J. R., 1999, MNRAS, 304, 75

8. Mather J., et al., 1990, Ap.J., 354, L37

9. Smoot G., et al., 1992, Ap.J.,1992, 396, L1

10. Miller, A. et al. 1999, Ap.J., 524, L1

11. Torbet E., et al., 1999, Ap.J., 521, L79-L82

12. Mauskopf P., et al., 2000, Ap.J., 536, L59-L62

13. de Bernardis, P., et al. 2000, Nature, 404, 955-959

14. Hanany, S. et al., 2000, Ap.J., 545, L5-L9

15. Netterfield B., et al., 2001, submitted to Ap.J., astro-ph/0104460

16. Leitch E.M., et al., 2001, astro-ph/0104488

17. de Bernardis, P., et al. 2002, Ap.J. in press, astro-ph/0105296

18. Pryke C., et al., astro-ph/0104490

19. Piacentini F., et al., these proceedings; see also astro-ph/0105148

20. Masi S., et al., these proceedings; see also Ap.J, 553, L93-L96, 2001, (astro-ph/0101539)

21. Masi, S., et al., 'Scanning polarimeters for measurements of CMB polarization', these proceedings.

22. Hristov V.V., et al., these proceedings.

23. Bock., J., et al., these proceedings.

24. Pascale E. and Boscaleri A., these proceedings.

25. Miller, A. et al. 2001, astro-ph/0108030

26. Lee A., et al., 1999, in "3K cosmology", AIP Conf. Proc. 476; astro-ph/9903249

27. White M., et al., astro-ph/9912422

28. Hanany S., et al., 1999, MNRAS, astro-ph/9801291

29. Hivon E. et al., 2001, astro-ph/0105302

30. Borrill, J., in 3K Cosmology astro-ph/9911389

31. Natoli P., et al. 2001, submitted to Ap.J., astro-ph/0101252

32. Halverson N.W. et al. 2001, astro-ph/0104489

33. Lee A.T. et al. 2001, astro-ph/0104459

34. Schlegel D.J. et al. 1999, Ap.J. 500, 525.

35. Polenta G., et al., in preparation.

36. Belen Barreiro R., 1999, astro-ph/9907094.

37. Sunyaev, R.A. \& Zeldovich , Ya.B., 1970, Astrophysics and Space Science 7, 3-19

38. Peebles, P.J.E, and Yu J.T., 1970, Ap.J., 162, 815

39. Percival W.J., 2001, astro-ph/0105252

40. see e.g. Avelino P.P., Shellard E.P.S., Wu J.H.P., and Allen B. 1998, Ap.J., 507, L101.

41. see e.g. Durrer R., Kunz M., and Melchiorri A. 1998, Phys. Rev. D V59, 123005)

42. Bouchet F.R. et al. 2001, astro-ph/0005022

43. Lewis A., Stewart E., Lasenby A., astro-ph/9911176

44. Seljak, U. \& Zaldarriaga, M. 1996, Ap.J. 469, 437

45. Turok N., et al., 2001, astro-ph/0012141

46. Weinberg S., 2000 , astro-ph/0006276

47. Melchiorri A. and Griffiths L.M., astro-ph/0011147

48. Gewiser E., astro-ph/0105010

49. Burles, S., Nollett, K.M. \& Turner, M.S. 2000, astro-ph/0010171

50. Bond, J.R., Jaffe, A.H. \& Knox, L. 1998, Phys. Rev. D57, 2117

51. Knox L. 2000, http://flight.uchicago.edu/knox/radpack.html

52. Weinberg S., 1989, Rev. Mod. Phys., 61, 1 